\title{
Aligned and Graded Type-II Ruddlesden-Popper Perovskite Films for Efficient Solar Cells
}

J ian Qing, Xiaoke Liu, Mingjie Li, Feng Liu, Zhongcheng Yuan, Elizaveta Tiukalova, Zhibo Yan, Martial Duchamp, Shi Chen, Yuming Wang, Sai Bai, J un-Ming Liu, Henry J. Snaith, Chun-Sing Lee, Tze Chien Sum and Feng Gao

The self-archived postprint version of this journal article is available at Linköping University Institutional Repository (DiVA):

http:// urn.kb.se/ resolve?urn=urn:nbn:se:liu:diva-152101

N.B.: When citing this work, cite the original publication.

Qing, J., Liu, X., Li, M., Liu, F., Yuan, Z., Tiukalova, E., Yan, Z., Duchamp, M., Chen, S., Wang, Y., Bai, S., Liu, J., Snaith, H. J., Lee, C., Sum, T. C., Gao, F., (2018), Aligned and Graded Type-II RuddlesdenPopper Perovskite Films for Efficient Solar Cells, ADVANCED ENERGY MATERIALS, 8(21), 1800185. https:// doi.org/ 10.1002/ aenm.201800185

Original publication available at:

https:// doi.org/ 10.1002/ aenm.201800185

Copyright: Wiley (12 months)

http:// eu.wiley.com/WileyCDA/ 


\section{WILEY-VCH}

DOI: 10.1002/((please add manuscript number))

Article type: Communication

\section{Aligned and Graded Type-II Ruddlesden-Popper Perovskite Films for Efficient Solar Cells}

Jian Qing, Xiao-Ke Liu, Mingjie Li, Feng Liu, Zhongcheng Yuan, Elizaveta Tiukalova, Zhibo Yan, Martial Duchamp, Shi Chen, Yuming Wang, Sai Bai, Jun-Ming Liu, Henry J. Snaith, Chun-Sing Lee*, Tze Chien Sum* and Feng Gao*

Dr. J. Qing, Prof. C. S. Lee

Center of Super-Diamond and Advanced Films (COSDAF) and Department of Chemistry, City University of Hong Kong, Hong Kong SAR, P. R. China.

E-mail: apcslee@cityu.edu.hk

Dr. J. Qing, Dr. X. K. Liu, Z. Yuan, Dr. Z. Yan, Y. Wang, Dr. S. Bai, Dr. F. Gao

Department of Physics, Chemistry and Biology (IFM), Linköping University, Linköping 58183, Sweden.

E-mail: fenga@ifm.liu.se

Dr. M. Li, Dr. S. Chen, Dr. T. C. Sum

Division of Physics and Applied Physics, School of Physical and Mathematical Sciences, Nanyang Technological University, 21 Nanyang Link, Singapore 637371, Singapore.

E-mail: tzechien@ntu.edu.sg

Prof. F. Liu

Department of Physics and Astronomy, and Collaborative Innovation Center of IFSA (CICIFSA), Shanghai Jiao Tong University, Shanghai 200240, P. R. China.

E. Tiukalova, Dr. M. Duchamp

School of Materials Science and Engineering, Nanyang Technological University, 50 Nanyang Avenue, Singapore 639798, Singapore.

Dr. Z. Yan, Prof. J.-M. Liu

Laboratory of Solid State Microstructures and Innovation Center of Advanced Microstructures, Nanjing University, Nanjing 210093, P. R. China

Dr. S. Chen

Institute of Material Research and Engineering, A*-STAR, 2 Fusionopolis Way, Singapore 138634, Singapore.

Dr. S. Bai, Prof. H. J. Snaith, Dr. F. Gao

Department of Physics, Clarendon Laboratory, University of Oxford, Parks Road, Oxford OX1 3PU, United Kingdom. 


\section{WILEY-VCH}

Keywords: layered perovskite, solar cell, additive, charge separation, 2D

\section{Abstract}

Recently, Ruddlesden-Popper perovskites (RPPs) have attracted increasing interests due to their promising stability. However, the efficiency of solar cells based on RPPs is much lower than that based on three-dimensional perovskites, mainly attributed to their poor charge transport. Herein, we present a simple yet universal method for controlling the quality of RPP films by a synergistic effect of two additives in the precursor solution. RPP films achieved by this method show a) high-quality with uniform morphology, enhanced crystallinity and reduced density of sub band gap states, b) vertically oriented perovskite frameworks that facilitate efficient charge transport, and c) type-II band alignment that favours self-driven charge separation. Consequently, we achieve a hysteresis-free RPP solar cell with a power conversion efficiency exceeding $12 \%$, which is much higher than that of the control device (1.5\%). Our findings will spur new developments in the fabrication of high quality, aligned and graded RPP films essential for realizing efficient and stable perovskite solar cells.

Organic-inorganic halide perovskites, such as $\mathrm{MAPbI}_{3}\left(\mathrm{MA}=\mathrm{CH}_{3} \mathrm{NH}_{3}\right), \mathrm{FAPbI}_{3}(\mathrm{FA}=$ $\left.\mathrm{CH}\left(\mathrm{NH}_{2}\right)_{2}\right)$ and their mixed cation analogues, have emerged as promising light absorbers for solar cells due to their superior photophysical properties and promise for low-cost solution processability. ${ }^{[1-4]}$ Perovskite solar cells have experienced tremendous development in the past few years with power conversion efficiency (PCE) rapidly increased to over $20 \%{ }^{[5,6]}$ Besides efficiency, another critical factor for practical application of perovskite solar cells is stability.

Recently, Ruddlesden-Popper perovskites (RPPs) have been reported to have promising stability. ${ }^{[7-9]}$ The RPPs can be structurally derived from their 3D (three-dimensional) counterparts with alternating organic ammonium layers and perovskite layers, giving the general formula of $\left(\mathrm{RNH}_{3}\right)_{2} \mathrm{~A}_{n-1} \mathrm{M}_{n} \mathrm{X}_{3 n+1}$, where $n$ represents the number of perovskite layers, $\mathrm{RNH}_{3}$ is the organic spacer, and the A (small cation), $\mathrm{M}$ (divalent metal cation) and $\mathrm{X}$ (halide 


\section{WILEY-VCH}

anion) form the perovskite framework. ${ }^{[10,11]}$ The superior stability of RPPs can be attributed to the hydrophobic property of the organic spacer, which inhibits water molecules from penetrating and attacking the inorganic layers.

Previous studies reported that RPPs exhibit enhanced stability but at the cost of reduced efficiency due to lower crystallinity and poor charge transport. ${ }^{[7,12-14]}$ The insulating nature of the organic layers is expected to inhibit the transport of charge carriers. Recently, a novel hot-casting technique has been proposed to fabricate high-quality RPP films with preferred growth orientation to favour efficient charge transport, giving a high PCE of $12.51 \%$ in $(\mathrm{BA})_{2}(\mathrm{MA})_{3} \mathrm{~Pb}_{4} \mathrm{I}_{13}(\mathrm{BA}=n$-butylammonium $)$ based solar cells. ${ }^{[8]}$ By doping a small amount of cesium cation $\left(\mathrm{Cs}^{+}\right)$into the RPP, an enhanced PCE of $13.7 \%$ is realized. ${ }^{[15]}$ However, the hot-casting technique requires precise temperature control of the substrates, hindering its application in large-area manufacture. ${ }^{[16]}$ Very recent studies on tin-based RPP films indicate that the preferential orientation can be controlled by precursor solvents. ${ }^{[17]}$ Similar results are found in lead-based RPP film (BA) $)_{2}(\mathrm{MA})_{4} \mathrm{~Pb}_{5} \mathrm{I}_{16}$, which is highly oriented when deposited from DMF (dimethylformamide)/DMSO (dimethylsulfoxide) mixtures using the hot-casting method, resulting in improved PCE of $10 \%$ in solar cells. ${ }^{[18]}$ In addition, the use of iso-BA instead of BA significantly improves the crystallinity and out-of-plane preferential orientation of RPP films. ${ }^{[16]}$ The iso-BA based RPP solar cells prepared at room temperature and using hot-casting method deliver PCEs of $8.82 \%$ and $10.63 \%$, respectively, however, showing significant hysteresis. ${ }^{[16]}$ Therefore, it is essential to develop a facile method to achieve high-quality RPP films for efficient and hysteresis-free solar cells.

In this work, we report a simple and universal method for growing high-quality RPP films, which results in hysteresis-free solar cells with a high PCE over $12 \%$ and excellent reproducibility. These high-quality films are achieved by incorporating DMSO and $\mathrm{CH}_{3} \mathrm{NH}_{3} \mathrm{Cl}$ (MACl) additives into precursor solution, followed by one-step spin-coating and solvent annealing processes. We systematically study the synergistic effects of DMSO and 


\section{WILEY-VCH}

MACl additives on controlling the crystallization of RPPs. We find that the additives can greatly improve the film quality of RPPs, leading to uniform morphology, enhanced crystallinity, and reduced energetic disorder. In addition, the resulting films show preferential orientation with their perovskite frameworks perpendicular to the substrate, which we assume is responsible for facilitating efficient carrier transport. Furthermore, they show graded distribution of multiple RPP phases with type-II band alignment, which is favourable for self-driven charge separation. Our method paves the way for further development of high-quality RPP films for efficient optoelectronic devices.

$(\mathrm{PEA})_{2}(\mathrm{MA})_{3} \mathrm{~Pb}_{4} \mathrm{I}_{13}\left(\mathrm{PEA}=\mathrm{C}_{6} \mathrm{H}_{5}\left(\mathrm{CH}_{2}\right)_{2} \mathrm{NH}_{3}\right)$ is used as the RPP in this study. The precursor solutions were prepared by dissolving $\mathrm{PbI}_{2}$, MAI and PEAI (4:3:2 molar ratio) in anhydrous DMF and DMSO with different volume ratios. For the chlorine-containing additive, $\mathrm{PbCl}_{2}$ and MAI (1:2 molar ratio) were added. Since in situ MACl was supposed to be formed through the reaction of $\mathrm{PbCl}_{2}+2 \mathrm{MAI}=\mathrm{PbI}_{2}+2 \mathrm{MACl}$, of which the effect was proved to be similar to that of using MACl directly, here we use MACl to describe the chlorine-containing additive for convenience. Morphologies of the RPP films are investigated by tapping-mode atomic force microscopy (AFM) and scanning electron microscopy (SEM) as shown in

Figure 1. These films show uniform morphologies with almost full coverage, suggesting the advantage of RPPs on forming compact and smooth films. The control film consists of small crystals with root-mean-squared (RMS) roughness of $15.2 \mathrm{~nm}$. RPP films with DMSO, MACl or both additives show enhanced crystallinity and reduced RMS roughness of 9.9, 10.9 and $7.9 \mathrm{~nm}$, respectively. However, the film with 6.7 vol\% DMSO has pinholes on the surface and cavities at RPP/PEDOT:PSS interface (Figure $1 \mathrm{~b}$ and c). The film with $10 \mathrm{~mol} \% \mathrm{MACl}$ shows just slightly increased crystal size, still having lots of grain boundaries. Interestingly, the optimized film with both 6.7 vol\% DMSO and $10 \mathrm{~mol} \% \mathrm{MACl}$ additives exhibits large and uniform single-crystalline-like perovskite grains without grain boundaries observed in the horizontal direction (Figure 1c). Thus, the transport of charge carriers is expected to be 


\section{WILEY-VCH}

enhanced along the vertical direction without grain boundary interference. AFM and SEM images in Figure S1 and S2 show the impact on the morphologies of the RPP films for different amounts of additives. As a rule of thumb, both DMSO and MACl increase the crystallinity and grain size but excess of additives could result in films with pinholes and rough surfaces. The two additives show synergistic effects on the morphologies of the RPP films. It is interesting to note that the RPP film with 6.7 vol\% DMSO shows pinholes which can be avoided by adding 5-10 mol\% MACl. However, excess MACl causes more pinholes (Figure S1c).

As shown in Figure 2a, X-ray diffraction (XRD) measurements are conducted to further investigate the crystallinity and orientation of the RPP films. Interestingly, no evenly spaced reflections are observed with $2 \theta$ below $14^{\circ}$. In contrast, strong diffraction signals are found at $2 \theta=14.2^{\circ}$ and $28.5^{\circ}$, which correspond to the (110) and (220) lattice planes, respectively ${ }^{[7]}$. Compared with the control film, the films with additive(s) yield remarkably enhanced diffraction intensities of peaks at $14.2^{\circ}$ and $28.5^{\circ}$. In addition, the full-width at half-maximum (FWHM) of the (110) plane is greatly reduced from $1.29^{\circ}$ for the control film to $0.19^{\circ}, 0.25^{\circ}$, $0.16^{\circ}$ for the films with $6.7 \mathrm{vol} \% \mathrm{DMSO}, 10 \mathrm{~mol} \% \mathrm{MACl}$ and both additives, respectively. These results suggest that the additives greatly favor the preferential crystal growth of (110) plane parallel to the substrate surface as well as improve crystallinity.

Grazing-incidence wide-angle X-ray scattering (GIWAXS) measurements are performed to further determine the orientation of the RPP films (Figure $2 b$ and c). ${ }^{[19]}$ The control film displays Debye-Scherrer rings at specific q values, suggesting mainly random crystal orientation within the polycrystalline film (Figure 2b). On the contrary, for the optimized film with both additives, the rings are absent, and intense, sharp and discrete Bragg spots are observed along the same q position (Figure 2c), indicating highly oriented crystal grains ${ }^{[8,20]}$. Indexing the observed Bragg peaks confirms that the crystal domains are oriented with their (110) planes parallel to the substrate surface. Such alignment indicates that the inorganic 


\section{WILEY-VCH}

layers are perpendicular to the substrate (Figure 2f). We presume that this will enable the formation of continuous charge-transport channels, and thus allow efficient charge transport in the vertical direction without impediment by the insulating organic layers.

The UV-vis absorption spectra of the RPP films in Figure 2d show an absorption onset at $\sim 750 \mathrm{~nm}$. In contrast to the control film, additional higher-energy peaks at around 514, 566, 602 and $635 \mathrm{~nm}$ appear in the RPP films with additive(s), which can be assigned to the excitonic absorption of (PEA) $)_{2}(\mathrm{MA})_{n-1} \mathrm{~Pb}_{n} \mathrm{I}_{3 n+1}$ with $n=1,2,3$ and 4 respectively. Thus, the films appear to be a mixture of multiple RPP phases with different $n$ values, which is consistent with previous reports ${ }^{[7,20-22]}$. The background apparent absorption at low energy for the DMSO-only film, can be attributed to film roughness and specifically cavities inside the film (Figure 1c), which can induce light scattering. To examine the uniformity of the optimized film with both additives, photoluminescence (PL) spectra are conducted with excitation wavelength at $400 \mathrm{~nm}$ from both front side (perovskite film side) and back side (substrate side). A dominant emission peak at $\sim 760 \mathrm{~nm}$ (attributed to large- $n$ RPPs) is observed for both excitations. However, back excitation shows additional peaks at higher energy, which can be attributed to the emission from the small-n RPP phases. Considering the shallow penetration depth $(\sim 60 \mathrm{~nm})$ under UV light one-photon excitation due to perovskite’s large absorption coefficient, ${ }^{[23]}$ only the surface of the perovskite film is excited. The difference of PL spectra between front and back excitations suggests that the small-n RPP phases prefer to locate at the bottom whereas the large-n RPP phases locate at the top of the film as illustrated in Figure 2f. Thus, we postulate that the film with both additives has vertically oriented and graded RPP phases.

To further confirm that a graded composition exists in the optimized film, we perform cross-sectional scanning transmission electron microscope (STEM) investigations (Figure 3a) and energy-dispersive X-ray spectroscopy (EDS) elemental mapping (Figure 3b). The high-angle annular-dark-field (HAADF) STEM image shows the increase of the average 


\section{WILEY-VCH}

atomic number from the bottom to the top of the perovskite film, suggesting the gradually increases of the $n$ values of the optimized RPP film from the bottom to the top. The bright field (BF) STEM image clearly shows colour change from the bottom to the top of the perovskite film, suggesting the increase in crystallinity toward the top surface, where the larger crystalline parts may correspond to larger- $n$ RPP phases. Both I and Pb elemental composition measured by EDS (Figure 3b) are found to show graded distributions with more I and $\mathrm{Pb}$ located at the top than bottom in agreement with the HAADF-STEM image. These observations are consistent with the results discussed above.

Transient absorption (TA) measurements are conducted to further confirm the graded phase distribution and examine the charge carrier separation processes in such optimized RPP film. Figure 3c shows time- and wavelength-dependent TA images under $400 \mathrm{~nm}$ front and back excitations, respectively. The negative photo-induced changes in absorption ( $-\Delta \mathrm{A})$ (i.e., photobleaching, $\mathrm{PB}$ ) indicate state filling of specific optical transitions. Under front excitation, there is one main PB peak at $~ 740 \mathrm{~nm}$ corresponding to the large- $n$ RPPs, associated with several very weak PB peaks from small-n RPPs. In contrast, upon back excitation, initially two PB peaks corresponding to $n=2$ and $n=3$ RPPs are observed. This is again consistent with the PL and STEM results that the $n$ values of RPP increase from the substrate side to the top. Furthermore, in contrast with the fast PB build-up of $n \geq 10$ RPP upon front excitation, the PB intensity of $n \geq 10$ RPP increases slowly under back excitation (Figure 3d). In addition, as shown in Figure 3e, a slow rise in lifetime of $\sim 450$ ps is observed for PB of large- $n$ ( $n \geq$ 10) RPPs, which agrees well with the relaxation time of PB peak of small- $n(n=2)$ RPPs. These results imply electron transfer from small- $n(n=2,3)$ RPPs at bottom to large- $n$ ( $n \geq$ 10) RPPs at surface of the film. A hole-transfer from surface large- $n$ RPPs to the bottom $n=2$ RPP with life time of $\sim 400$ ps is also observed (Figure S3). Interestingly, such phenomenon is not observed in the control film (Figure S4). 


\section{WILEY-VCH}

To gain deeper insights into the charge transfer/separation process and distinguish whether the electron- or hole-transfer from bottom to surface, the carrier-dynamics of such RPP film interfaced with [6,6]-phenyl-C61-butyric acid methylester (PCBM, electron-extraction layer) or 2,2,7,7-tetrakis(N,N-di-p-methoxyphenyl-amine) 9,9-spirobifluorene] (Spiro-OMeTAD, hole-extraction layer) at the surface are also probed. As shown in Figure 3f, upon back excitation for the RPP/PCBM bilayer film, the slow PB rise originating from large- $n$ ( $n \geq 10)$ RPPs at the surface disappears and the PB intensity is reduced and recovered much faster. This indicates that the PCBM removes the charges transferred from small- $n$ ( $n=2,3)$ RPPs at the bottom. In contrast, for the RPP/Spiro-OMeTAD bilayer film, the slow PB rise remains even with Spiro-OMeTAD at the surface, thus indicating that the holes generated at the bottom cannot diffuse to the top surface.

These observations of self-driven charge separation process are also supported by band alignment of RPPs with small and large $n$ values. The energy levels (as illustrated in Figure 3g) are determined by using linear-absorption and ultraviolet photoelectron spectroscopy (UPS) measurements (Figure S5). Figure S5a shows that the absorption bands of RPPs with low (2:9) and high (2:1) PEA:MA ratios that correspond well with PB peaks of small- $n(n=2$, 3) and large- $n$ ( $n \geq 10)$ RPPs (Figure 3c), thus they are representative of the properties of RPPs at the bottom and the top surface of the film, respectively. As shown in Figure 3g, the small- $n$ and large- $n$ RPPs form a type-II band alignment, favouring self-driven charge separation, which is consistent with the overall charge transport for inverted structure, thus benefiting for inverted device performance.

Photovoltaic performance of these RPP films are investigated with a device structure of ITO/PEDOT:PSS (40 nm)/RPP (330 nm)/PCBM (50 nm)/BCP (5 nm)/Ag (70 nm). Figure 4a shows the $\mathrm{J}-\mathrm{V}$ curves of the most representative devices, which are close to the average results obtained for each condition. The control device based on the RPP film without 


\section{WILEY-VCH}

additives shows a very low short-circuit current density $\left(\mathrm{J}_{\mathrm{sc}}\right)$ of $2.3 \mathrm{~mA} \mathrm{~cm}{ }^{-2}$, resulting in a poor PCE of $1.5 \%$. The addition of $\mathrm{MACl}$ or DMSO remarkably increases the $\mathrm{J}_{\mathrm{sc}}$ and open-circuit voltage $\left(\mathrm{V}_{\text {oc }}\right.$ ) (Figure 4a and $\left.\mathrm{S} 6\right)$. As shown in Figure S6a, the devices based on the (PEA) $)_{2}(\mathrm{MA})_{3} \mathrm{~Pb}_{4} \mathrm{I}_{13}$ films with 6.7 vol\% DMSO show clear improved $\mathrm{J}_{\mathrm{sc}}$ and $\mathrm{V}_{\mathrm{oc}}$ upon addition of MACl. Specifically, the $\mathrm{J}_{\mathrm{sc}}$ increases from 9.3 to $14.5 \mathrm{~mA} \mathrm{~cm}{ }^{-2}$ when the amount of MACl ranges from 0 mol\% to $10 \mathrm{~mol} \%$ (Table $\mathrm{S} 1$ ). The $\mathrm{J}_{\mathrm{sc}}$ drops rapidly with $\mathrm{MACl}$ exceeding 10 mol\%, which can be assigned to the inferior morphology of the RPP films (Figure S1). Similar trend can be observed on the $\mathrm{V}_{\text {oc }}$, which reaches its maximum of $1.16 \mathrm{~V}$ at $20 \mathrm{~mol} \% \mathrm{MACl}$ (Table S1). For the devices with $10 \mathrm{~mol} \% \mathrm{MACl}$ and different DMSO contents, both the $\mathrm{V}_{\text {oc }}$ and $\mathrm{J}_{\mathrm{sc}}$ increase remarkably. However, excess DMSO leads to decreased $\mathrm{J}_{\mathrm{sc}}$ and FF (fill factor). In consistent with the observation of morphologies, the DMSO and MACl have a synergistic effect on the device performance. As shown in Figure 4a, the RPP film with both additives shows the highest performance. The significant performance improvement can be ascribed to the formation of uniform and smooth RPP films with enhanced crystallinity and preferred orientation. In addition, as discussed above, the perpendicular growth of inorganic layers leads to efficient charge transport and the graded distribution of RPP phases promotes self-driven charge separation.

In addition, we believe the higher performance of the RPP film with additives can also be assigned to reduced defects and energetic disorder with suppressed charge recombination. We investigate the sub-bandgap states of the RPP films by Fourier transform photocurrent spectroscopy (FTPS) on complete solar cells. Generally, the density of sub-bandgap states can be obtained by fitting the exponential rise in the external quantum efficiency (EQE) spectra below the band gap, known as the Urbach tail, to derive the Urbach energy $\left(E_{\mathrm{u}}\right)$. As shown in Figure S7, the optimized film with both additives shows remarkably lower $E_{\mathrm{u}}$ of $15.7 \mathrm{meV}$ compared to $21.8 \mathrm{meV}$ for the control film without additives, indicating reduced sub band gap states $^{[24,25]}$. 


\section{WILEY-VCH}

It is important to note that the $\mathrm{V}_{\text {oc }}$ here $(1.16 \mathrm{~V})$ is significantly higher than that $(0.8-1.0$ V) which is typically achieved for the 3D perovskite (MAPbI 3 ) with the same device structure $^{[26,27]}$, suggesting RPPs as promising candidates for high-photovoltage devices. In addition, the devices show excellent reproducibility due to facile fabrication and precise control of the optimized ratios of additives. Figure $4 \mathrm{~b}$ gives the statistical distribution of the PCEs based on 50 devices, giving an average PCE of 10.7\% and the highest PCE of 12.1\%. Detailed performance statistics are shown in Figure S8. Figure 4c presents the J-V characteristics of the champion device, which delivers a high $\mathrm{V}_{\text {oc }}$ of $1.16 \mathrm{~V}$, a $\mathrm{J}_{\mathrm{sc}}$ of $14.7 \mathrm{~mA}$ $\mathrm{cm}^{-2}$, an FF of 0.71 , and a PCE of $12.1 \%$.

The external quantum efficiency (EQE) spectrum and integrated $\mathrm{J}_{\mathrm{sc}}$ are plotted in Figure 4d. The EQE spectrum matches well with the optical absorption and the integrated $\mathrm{J}_{\text {sc }}$ of 14.3 $\mathrm{mA} \mathrm{cm}{ }^{-2}$ is in good agreement with that derived from $\mathrm{J}-\mathrm{V}$ measurement. Figure 4e shows the J-V curves under different scan rates and scan directions. Negligible photocurrent hysteresis can be observed in our devices, which is in good agreement with previous reports using similar high-quality RPP films ${ }^{[8,26]}$. We also measure the stabilized photocurrent and steady efficiency at the maximum power output point $(0.94 \mathrm{~V})$, as shown in Figure 4f. The photocurrent density immediately reaches $12.9 \mathrm{~mA} \mathrm{~cm}^{-2}$ upon light on and maintains constant under continuous illumination, resulting in a stabilized PCE of 12.1\%.

To prove our method is universal for different types of RPPs, we extend our study to FA based RPPs $\left((\mathrm{PEA})_{2}(\mathrm{FA})_{3} \mathrm{~Pb}_{4} \mathrm{I}_{13}\right.$ and $\left.(\mathrm{PEA})_{2}\left(\mathrm{Cs}_{0.15} \mathrm{FA}_{0.85}\right)_{3} \mathrm{~Pb}_{4} \mathrm{I}_{13}\right)$. In 3D perovskites, by replacing the MA cation with formamidinium (FA) cation, $\mathrm{FAPbI}_{3}$ was reported to have extended light harvesting capability and better thermal stability. ${ }^{[28,29]}$ However, the black perovskite $\mathrm{FAPbI}_{3}$ tends to convert into yellow non-perovskite phase in the presence of moisture. By partially substituting FA with $\mathrm{Cs}$, the mixed cation perovskite $\left(\mathrm{Cs}_{\mathrm{x}} \mathrm{FA}_{1-\mathrm{x}} \mathrm{PbI}_{3}\right)$ was demonstrated to show enhanced performance and stability. ${ }^{[30,31]}$ Interestingly, the control film of $(\mathrm{PEA})_{2}(\mathrm{FA})_{3} \mathrm{~Pb}_{4} \mathrm{I}_{13}$ without additives contains yellow $\mathrm{FAPbI}_{3}$, as revealed by the 


\section{WILEY-VCH}

XRD (Figure S9a), as well as more (PEA) $)_{2}(\mathrm{FA}) \mathrm{Pb}_{2} \mathrm{I}_{7}(n=2)$ phase (Figure S9b). Additives successfully suppress the formation of the yellow phase. Efficient devices with best PCEs about $12 \%$ are also achieved for both FA and CsFA based RPPs (Figure S9c and Table S3), which are much higher than those of the control devices (PCE of 1\%). These results suggest the universality of our method.

In addition to high efficiency, the long-term stability of perovskite solar cells is a major concern for commercialization. A comparative study on moisture stability is conducted by exposing the perovskite films to ambient air with 70\% relative humidity and XRD spectra are recorded to monitor the degradation. As shown in Figure S10a, major peaks of $\mathrm{MAPbI}_{3}$ perovskite at $14.2^{\circ}$ and $28.4^{\circ}$ completely disappear after exposure to air for 10 days, while a new peak at $12.8^{\circ}$ is detected, which can be assigned to $\mathrm{PbI}_{2}$. The inset photographs in Figure S10a shows the color of film changes from black to yellow, indicating the severe decomposition of $\mathrm{MAPbI}_{3}$ to $\mathrm{PbI}_{2}$ because of the loss of $\mathrm{MA}$ cation. In contrast, the $(\mathrm{PEA})_{2}(\mathrm{MA})_{3} \mathrm{~Pb}_{4} \mathrm{I}_{13}$ film with both additives exhibited much improved stability, showing barely color change and the XRD patterns just show slightly decrease on intensity without emerging new peaks (Figure S10b). The remarkable stability of the RPPs can be attributed to the hydrophobicity of the large organic cation, which shields the inner perovskite layers from moisture.

We further evaluate the stability of complete devices without encapsulation in ambient air with $45 \%$ relative humidity. Figure S10c shows the normalized photovoltaic parameters as a function of storage time. The reference devices based on $\mathrm{MAPbI}_{3}$ degrade dramatically, with a drop in PCE to near zero in 10 days. On the contrary, the (PEA) $2(\mathrm{MA})_{3} \mathrm{~Pb}_{4} \mathrm{I}_{13}$ based devices exhibit significantly improved stability, showing even a slightly increase on PCE in the first 10 days and maintaining $50 \%$ of their initial PCEs after storage for one month. The initial performance improvement arising from the increase of $\mathrm{J}_{\mathrm{sc}}$ might be explained by moisture-induced crystallization and passivation process. ${ }^{[32,33]}$ The photograph of devices in 


\section{WILEY-VCH}

Figure S10d shows the color change at the edge of Ag electrode for the $\mathrm{MAPbI}_{3}$ based device, while the $(\mathrm{PEA})_{2}(\mathrm{MA})_{3} \mathrm{~Pb}_{4} \mathrm{I}_{13}$ based device remained almost unchanged visually. Besides the degradation of perovskites themselves, the stability of device is sensitive to many factors, such as interfacial layers and electrode. The hygroscopic nature of PEDOT:PSS could degrade the interfaces of PEDOT:PSS/ITO. ${ }^{[34]}$ PCBM was reported to degrade in ambient air through adsorption of oxygen or water. ${ }^{[35]}$ Even Ag electrode can be corroded to produce AgI. ${ }^{[36,37]}$ Thus, the organic interfacial layers and Ag electrode may limit the stability of our devices. In addition, the composition of the RPPs is also a key factor determining the stability of the films and devices. As shown in Figure S11, the (PEA) $)_{2}(\mathrm{FA})_{3} \mathrm{~Pb}_{4} \mathrm{I}_{13}$ based film and device degrade rapidly in moisture condition. However, the (PEA) ${ }_{2}\left(\mathrm{Cs}_{0.15} \mathrm{FA}_{0.85}\right)_{3} \mathrm{~Pb}_{4} \mathrm{I}_{13}$ based film and device exhibit remarkably improved stability. Impressively, the PCE of the (PEA) $)_{2}\left(\mathrm{Cs}_{0.15} \mathrm{FA}_{0.85}\right)_{3} \mathrm{~Pb}_{4} \mathrm{I}_{13}$ based device maintains up to $90 \%$ after being exposed in ambient air with $45 \%$ relative humidity for 30 days. Further studies on robust interfacial, electrode materials, and composition management are anticipated to produce highly efficient RPP solar cells with practical durability. ${ }^{[38]}$

In summary, we demonstrate a novel approach to fabricate high-quality RPP films for efficient solar cells. The key lies in the synergistic effects of DMSO and MACl additives, which assist the crystallization of perovskites, leading to single-crystal-like RPP films with smooth surface, high crystallinity, and low defect densities. Spectroscopic and microscopic investigations reveal self-organization of type-II band alignment of the RPPs with different $n$ values, facilitating self-driven charge separation. In addition, the additives induce growth of vertically oriented perovskite frameworks, enabling efficient charge transport. Our work demonstrates the great potential of RPPs as a promising photovoltaic material, and also paves the way for further applications of RPPs in other optoelectronic devices.

\section{Supporting Information}




\section{WILEY-VCH}

Supporting Information is available from the Wiley Online Library or from the author.

\section{Acknowledgements}

J. Qing, X. K. Liu, and M. Li contribute equally to this work. This work is supported by the Research Grants Council of the Hong Kong Special Administrative Region, China (Project No. 11304115), the National Natural Science Foundation of China (No. 51473138), the joint NTU-LiU PhD programme on Materials- and Nanoscience, the Swedish Research Council VR (Grant No. 330-2014-6433), the European Commission Marie Skłodowska-Curie action (Grant No. INCA 600398 and No. 691210), and the Swedish Government Strategic Research Area in Materials Science on Functional Materials at Linköping University (Faculty Grant SFO-Mat-LiU no. 2009-00971). T.C.S. acknowledges the financial support from Nanyang Technological University start-up grant M4080514; the Ministry of Education Academic Research Fund Tier 1 grants RG101/15 and RG173/16, and Tier 2 grants MOE2014-T2-1-044, MOE2015-T2-2-015 and MOE2016-T2-1-034; and from the Singapore National Research Foundation through the Competitive Research Program NRF-CRP14-2014. X.K.L. would like to thank the VINNMER and Marie Skłodowska-Curie Fellowship (2016-02051) provided by Vinnova. We thank Quanzheng Tao (Linköping University) for assisting XRD measurements. The TEM measurement was performed at the Facility for Analysis, Characterization, Testing and Simulation (FACTS) in Nanyang Technological University, Singapore.

Received: ((will be filled in by the editorial staff))

Revised: ((will be filled in by the editorial staff)) Published online: ((will be filled in by the editorial staff))

\section{References}

[1] S. D. Stranks, G. E. Eperon, G. Grancini, C. Menelaou, M. J. P. Alcocer, T. Leijtens, L. M. Herz, A. Petrozza, H. J. Snaith, Science 2013, 342, 341.

[2] G. C. Xing, N. Mathews, S. Y. Sun, S. S. Lim, Y. M. Lam, M. Gratzel, S. Mhaisalkar, T. C. Sum, Science 2013, 342, 344.

[3] C. T. Zuo, H. J. Bolink, H. W. Han, J. S. Huang, D. Cahen, L. M. Ding, Adv. Sci. 2016, 3, 1500324.

[4] T. M. Brenner, D. A. Egger, L. Kronik, G. Hodes, D. Cahen, Nat. Rev. Mater. 2016, 1, 15007.

[5] W. S. Yang, J. H. Noh, N. J. Jeon, Y. C. Kim, S. Ryu, J. Seo, S. I. Seok, Science 2015, 348, 1234. 


\section{WILEY-VCH}

[6] M. Saliba, T. Matsui, K. Domanski, J. Y. Seo, A. Ummadisingu, S. M. Zakeeruddin, J. P. Correa-Baena, W. R. Tress, A. Abate, A. Hagfeldt, M. Gratzel, Science 2016, 354, 206.

[7] I. C. Smith, E. T. Hoke, D. Solis-Ibarra, M. D. McGehee, H. I. Karunadasa, Angew. Chem.-Int. Edit. 2014, 53, 11232.

[8] H. H. Tsai, W. Y. Nie, J. C. Blancon, C. C. S. Toumpos, R. Asadpour, B. Harutyunyan, A. J. Neukirch, R. Verduzco, J. J. Crochet, S. Tretiak, L. Pedesseau, J. Even, M. A. Alam, G. Gupta, J. Lou, P. M. Ajayan, M. J. Bedzyk, M. G. Kanatzidis, A. D. Mohite, Nature 2016, 536, 312.

[9] S. Chen, G. Q. Shi, Adv. Mater. 2017, 29, 1605448.

[10] C. C. Stoumpos, D. H. Cao, D. J. Clark, J. Young, J. M. Rondinelli, J. I. Jang, J. T. Hupp, M. G. Kanatzidis, Chem. Mater. 2016, 28, 2852.

[11] C. C. Stoumpos, C. M. Soe, H. Tsai, W. Nie, J. C. Blancon, D. H. Cao, F. Liu, B. Traore, C. Katan, J. Even, A. D. Mohite, M. G. Kanatzidis, Chem. 2017, 2, 427-440.

[12] D. H. Cao, C. C. Stoumpos, O. K. Farha, J. T. Hupp, M. G. Kanatzidis, J. Am. Chem. Soc. 2015, 137, 7843.

[13] Z. G. Xiao, R. A. Kerner, L. F. Zhao, N. L. Tran, K. M. Lee, T. W. Koh, G. D. Scholes, B. P. Rand, Nat. Photonics 2017, 11, 108.

[14] L. N. Quan, M. J. Yuan, R. Comin, O. Voznyy, E. M. Beauregard, S. Hoogland, A. Buin, A. R. Kirmani, K. Zhao, A. Amassian, D. H. Kim, E. H. Sargent, J. Am. Chem. Soc. 2016, 138, 2649.

[15] X. Zhang, X. D. Ren, B. Liu, R. Munir, X. J. Zhu, D. Yang, J. B. Li, Y. C. Liu, D. M. Smilgies, R. P. Li, Z. Yang, T. Q. Niu, X. L. Wang, A. Amassian, K. Zhao, S. Z. F. Liu, Energy Environ. Sci. 2017, 10, 2095.

[16] Y. N. Chen, Y. Sun, J. J. Peng, W. Zhang, X. J. Su, K. B. Zheng, T. Pullerits, Z. Q. Liang, Adv. Energy Mater. 2017, 7, 1700162. 


\section{WILEY-VCH}

[17] D. H. Cao, C. C. Stoumpos, T. Yokoyama, J. L. Logsdon, T. B. Song, O. K. Farha, M. R. Wasielewski, J. T. Hupp, M. G. Kanatzidis, ACS Energy Lett. 2017, 2, 982.

[18] C. M. M. Soe, W. Nie, C. C. Stoumpos, H. Tsai, J.-C. Blancon, F. Liu, J. Even, T. J. Marks, A. D. Mohite, M. G. Kanatzidis, Adv. Energy Mater. 2017, DOI: 10.1002/aenm.201700979.

[19] J. Schlipf, P. Muller-Buschbaum, Adv. Energy Mater. 2017, 7, 1700131.

[20] R. L. Milot, R. J. Sutton, G. E. Eperon, A. A. Haghighirad, J. M. Hardigree, L. Miranda, H. J. Snaith, M. B. Johnston, L. M. Herz, Nano Lett. 2016, 16, 7001.

[21] J. X. Liu, J. Leng, K. F. Wu, J. Zhang, S. Y. Jin, J. Am. Chem. Soc. 2017, 139, 1432.

[22] N. N. Wang, L. Cheng, R. Ge, S. T. Zhang, Y. F. Miao, W. Zou, C. Yi, Y. Sun, Y. Cao, R. Yang, Y. Q. Wei, Q. Guo, Y. Ke, M. T. Yu, Y. Z. Jin, Y. Liu, Q. Q. Ding, D. W. Di, L. Yang, G. C. Xing, H. Tian, C. H. Jin, F. Gao, R. H. Friend, J. P. Wang, W. Huang, Nat. Photonics 2016, 10, 699.

[23] M. A. Green, A. Ho-Baillie, H. J. Snaith, Nat. Photonics 2014, 8, 506.

[24] Z. H. Liu, J. N. Hu, H. Y. Jiao, L. Li, G. H. J. Zheng, Y. H. Chen, Y. Huang, Q. Zhang, C. Shen, Q. Chen, H. P. Zhou, Adv. Mater. 2017, 29, 1606774.

[25] B. D. Zhao, M. Abdi-Jalebi, M. Tabachnyk, H. Glass, V. S. Kamboj, W. Y. Nie, A. J. Pearson, Y. Puttisong, K. C. Godel, H. E. Beere, D. A. Ritchie, A. D. Mohite, S. E. Dutton, R. H. Friend, A. Sadhanala, Adv. Mater. 2017, 29.

[26] J. Qing, H. T. Chandran, Y. H. Cheng, X. K. Liu, H. W. Li, S. W. Tsang, M. F. Lo, C. S. Lee, ACS Appl. Mater. Interfaces 2015, 7, 23110.

[27] M. Qian, M. Li, X. B. Shi, H. Ma, Z. K. Wang, L. S. Liao, J. Mater. Chem. A 2015, 3, 13533.

[28] F. Wang, H. Yu, H. H. Xu, N. Zhao, Adv. Funct. Mater. 2015, 25, 1120.

[29] G. E. Eperon, S. D. Stranks, C. Menelaou, M. B. Johnston, L. M. Herz, H. J. Snaith, Energy Environ. Sci. 2014, 7, 982. 


\section{WILEY-VCH}

[30] J. W. Lee, D. H. Kim, H. S. Kim, S. W. Seo, S. M. Cho, N. G. Park, Adv. Energy Mater. 2015, 5, 1501310.

[31] M. Saliba, T. Matsui, J. Y. Seo, K. Domanski, J. P. Correa-Baena, M. K. Nazeeruddin, S. M. Zakeeruddin, W. Tress, A. Abate, A. Hagfeldt, M. Gratzel, Energy Environ. Sci. 2016, 9, 1989.

[32] C. Q. Ma, D. Shen, J. A. Qing, H. T. Chandran, M. F. Lo, C. S. Lee, ACS Appl. Mater. Interfaces 2017, 9, 14960.

[33] Y. G. Rong, X. M. Hou, Y. Hu, A. Y. Mei, L. F. Liu, P. Wang, H. W. Han, Nat. Commun. 2017, 8, 14555.

[34] T. A. Berhe, W. N. Su, C. H. Chen, C. J. Pan, J. H. Cheng, H. M. Chen, M. C. Tsai, L. Y. Chen, A. A. Dubale, B. J. Hwang, Energy Environ. Sci. 2016, 9, 323.

[35] Q. Y. Bao, X. J. Liu, S. Braun, M. Fahlman, Adv. Energy Mater. 2014, 4, 1301272.

[36] Y. Kato, L. K. Ono, M. V. Lee, S. H. Wang, S. R. Raga, Y. B. Qi, Adv. Mater. Interfaces 2015, 2, 1500195.

[37] J. W. Li, Q. S. Dong, N. Li, L. D. Wang, Adv. Energy Mater. 2017, 7, 1602922.

[38] J. B. You, L. Meng, T. B. Song, T. F. Guo, Y. Yang, W. H. Chang, Z. R. Hong, H. J. Chen, H. P. Zhou, Q. Chen, Y. S. Liu, N. De Marco, Y. Yang, Nat. Nanotechnol. 2016, $11,75$. 


\section{WILEY-VCH}

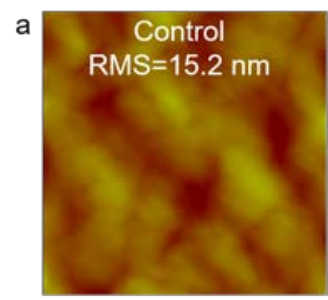

bontrol
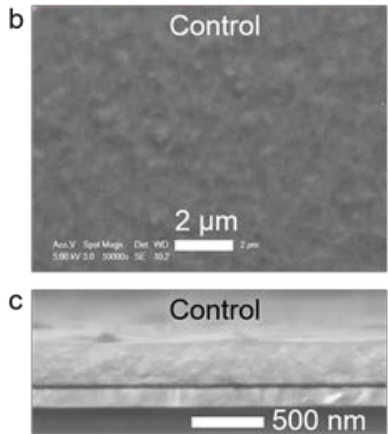
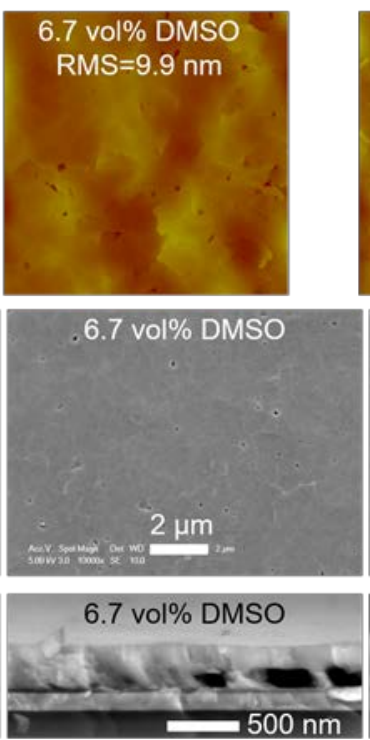
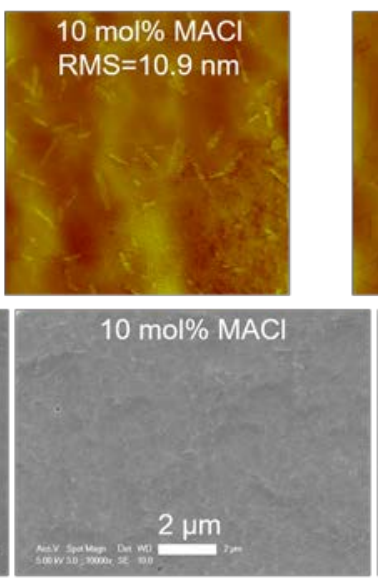

$10 \mathrm{~mol} \% \mathrm{MACl}$
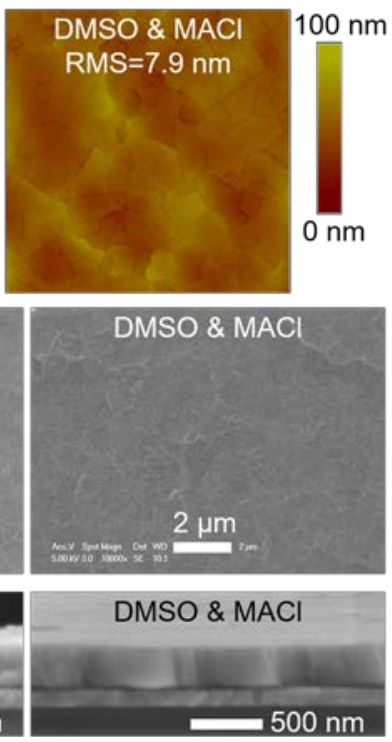

Figure 1. (a) AFM images (size: $5 \mu \mathrm{m} \times 5 \mu \mathrm{m}$ ), (b) top-view and (c) cross-sectional SEM images of the (PEA) $)_{2}(\mathrm{MA})_{3} \mathrm{~Pb}_{4} \mathrm{I}_{13}$ films without or with additive(s). The films are deposited on ITO/poly(3,4-ethylenedioxythiophene):poly(styrenesulfonate) (PEDOT:PSS) substrates.

a

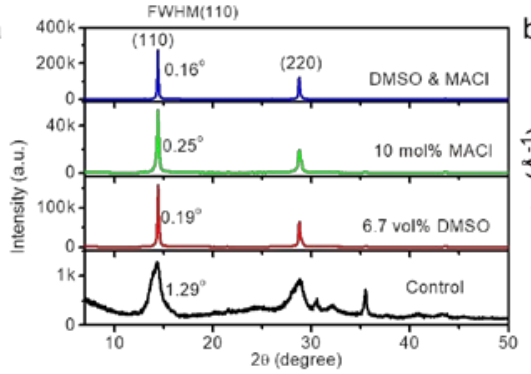

d

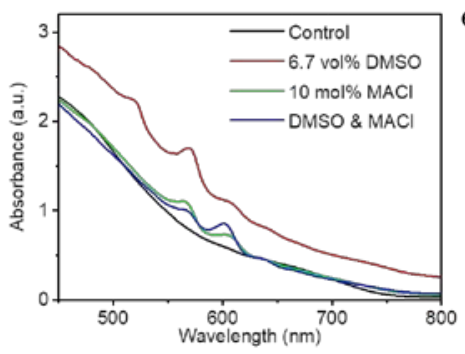

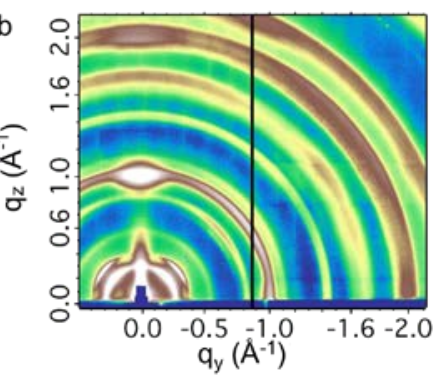

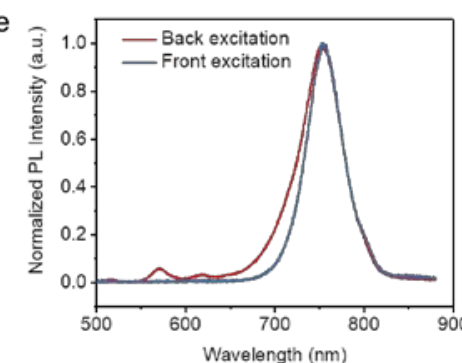

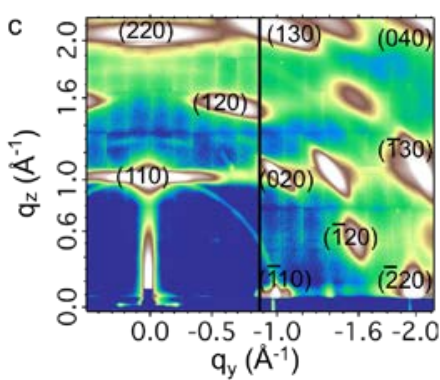

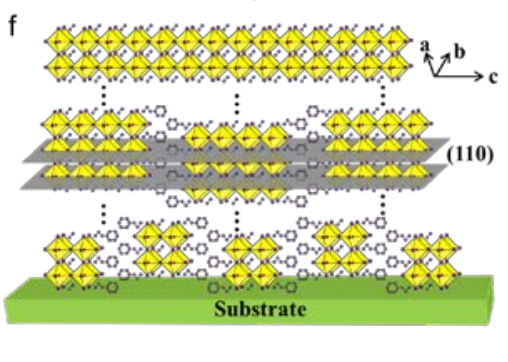

Figure 2. (a) XRD patterns for the (PEA) $)_{2}(\mathrm{MA})_{3} \mathrm{~Pb}_{4} \mathrm{I}_{13}$ films without or with different additives. GIWAXS patterns of the (PEA) $)_{2}(\mathrm{MA})_{3} \mathrm{~Pb}_{4} \mathrm{I}_{13}$ films (b) without additives and (c) with both additives. (d) Absorption spectra of the RPP films without and with different additives. (e) PL spectra of the RPP film with both additives excited from the front side and back side. (f) Schematic illustration of preferential crystal growth with (110) plane parallel to the substrate as well as graded multiple RPP phases. 
WILEY-VCH
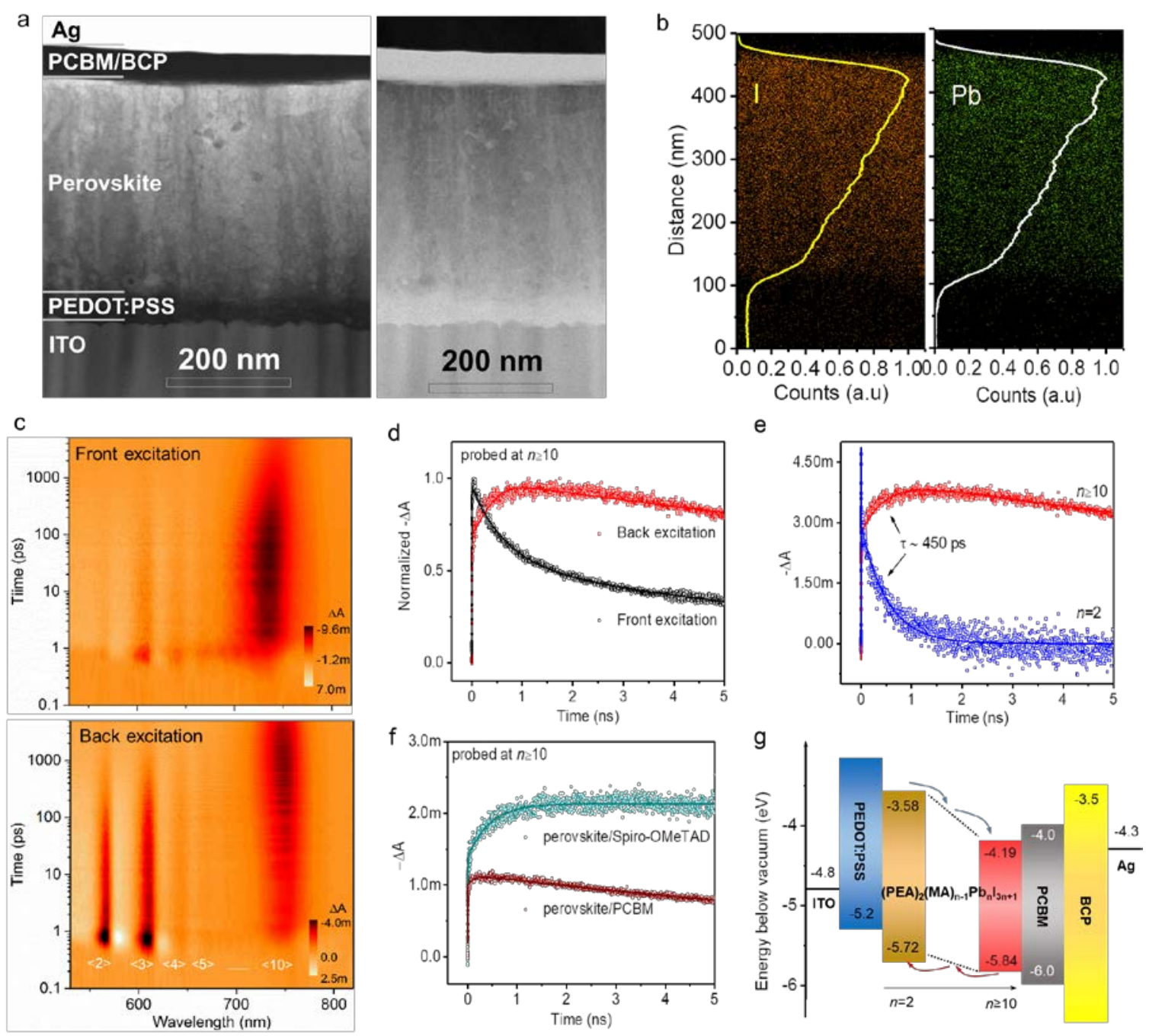

Figure 3. (a) Cross-sectional high-angle annular dark field (HAADF) (left) and bright field (BF) STEM images (right). (b) Elemental EDS maps showing the distributions of I and $\mathrm{Pb}$ for $(\mathrm{PEA})_{2}(\mathrm{MA})_{3} \mathrm{~Pb}_{4} \mathrm{I}_{13}$ film with DMSO and MACl additives. (c) Pseudocolour plot TA spectra of the RPP film with both two additives on glass excited from the top surface (upper panel) and from the bottom surface (lower panel). (d) Normalized TA dynamics probed at large- $n$ ( $n$ $\geq 10$ ) RPP phase (at around $740 \mathrm{~nm}$ ) under 400-nm excitation from the back (red) and front (black), respectively. (e) TA dynamics probed at small- $n(n=2)$ (blue) and large- $n(n \geq 10)$ (red) RPPs, respectively. (f) TA dynamics probed at large- $n(n \geq 10)$ RPPs in perovskite/PCBM and perovskite/Spiro-OMeTAD bilayers. Solid curves in (d), (e) and (f) are exponential decay fittings. (g) Energy level alignment of RPPs with $n=2$ and $n \geq 10$, and a schematic illustration of the charge transfer process in a type-II band alignment. 


\section{WILEY-VCH}

a
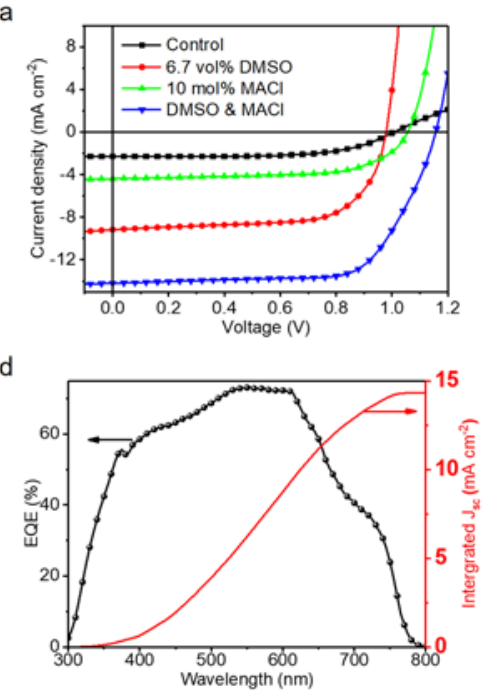

b
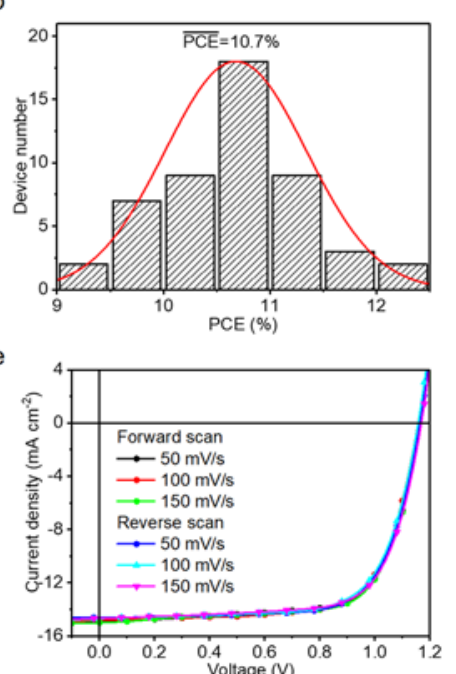

c
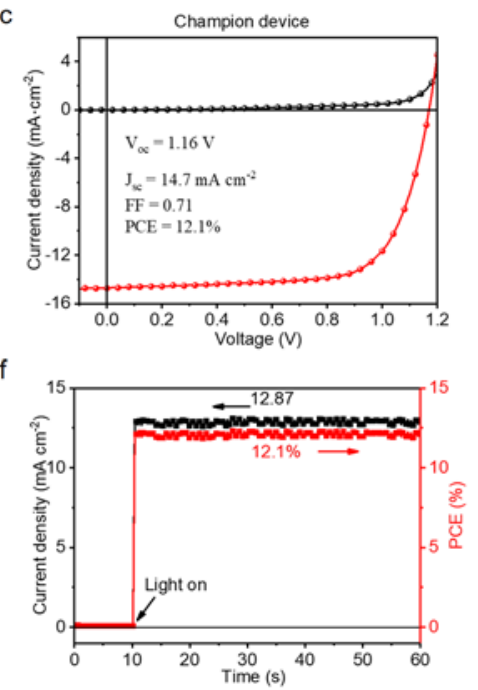

Figure 4. (a) $\mathrm{J}-\mathrm{V}$ curves of devices fabricated based on (PEA) $)_{2}(\mathrm{MA})_{3} \mathrm{~Pb}_{4} \mathrm{I}_{13}$ films without or with additive(s). (b) Histogram of PCEs based on (PEA) $)_{2}(\mathrm{MA})_{3} \mathrm{~Pb}_{4} \mathrm{I}_{13}$ films with both DMSO and MACl additives. (c) J-V curves of the champion device under dark and light conditions. (d) EQE and integrated $\mathrm{J}_{\mathrm{sc}}$ of the champion device. (e) $\mathrm{J}-\mathrm{V}$ curves of the device at different scan directions and scan rates. (f) Steady measurement at the maximum power output point of $0.94 \mathrm{~V}$. 